\title{
Overview the effect of statin therapy on dementia risk, cognitive changes and its pathologic change: a systematic review and meta-analysis
}

\author{
Xi-Chen Zhu, Wen-Zhuo Dai, Tao Ma \\ Department of Neurology, the Affiliated Wuxi No. 2 People's Hospital of Nanjing Medical University, Wuxi 214002, China \\ Contributions: (I) Conception and design: XC Zhu, T Ma; (II) Administrative support: T Ma; (III) Provision of study materials or patients: XC Zhu, \\ WZ Dai; (IV) Collection and assembly of data: XC Zhu; (V) Data analysis and interpretation: All authors; (VI) Manuscript writing: All authors; (VII) \\ Final approval of manuscript: All authors \\ Correspondence to: Dr. Xi-Chen Zhu, PhD; Tao Ma, MD, PhD. Department of Neurology, the Affiliated Wuxi No. 2 People's Hospital of Nanjing \\ Medical University, No. 68 Zhongshan Road, Wuxi 214002, China. Email: zxc890205@163.com; tmadoc@126.com.
}

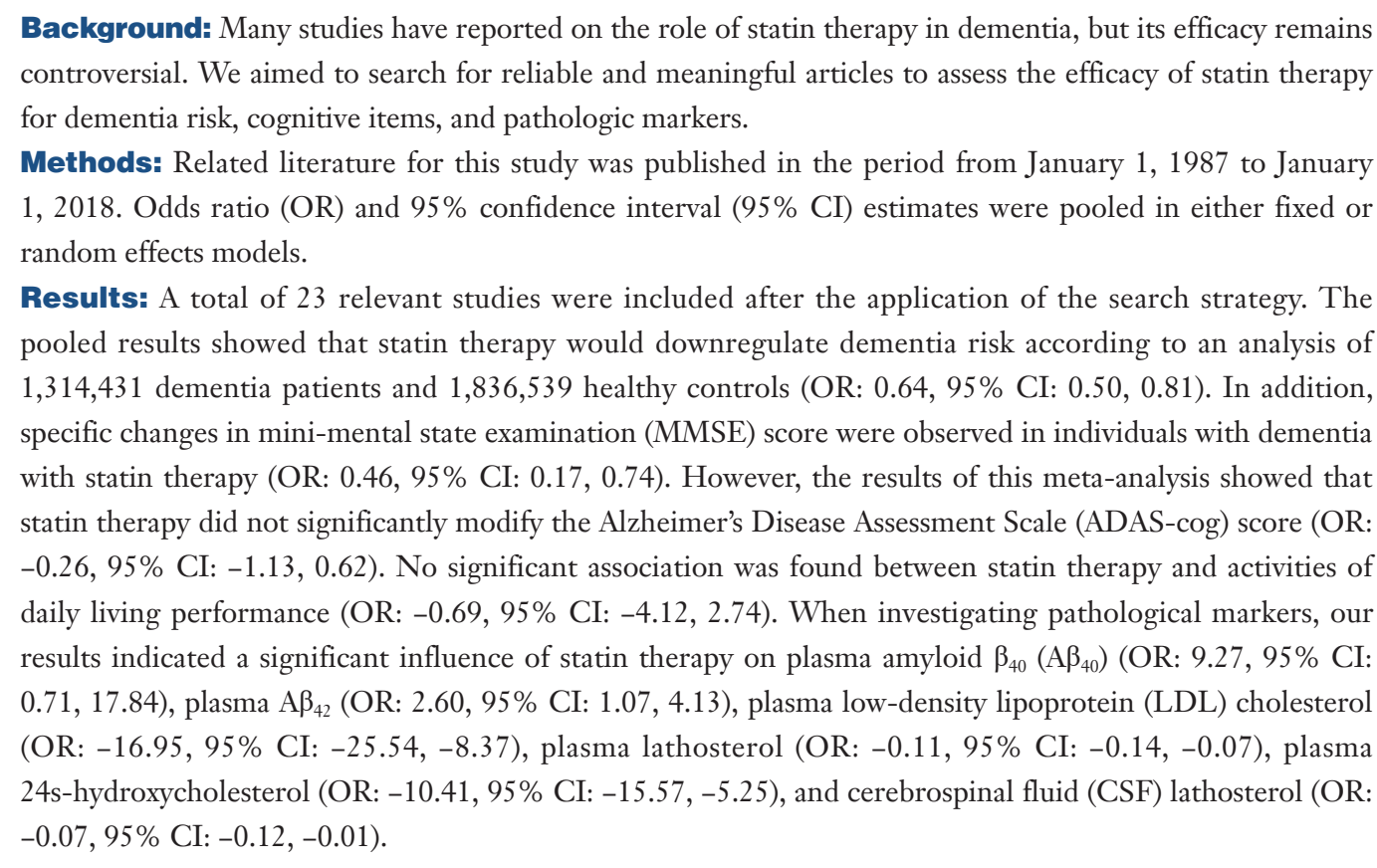

Conclusions: The available data indicate that statin therapy may reduce dementia risk, altering cognitive items and pathologic markers.

Keywords: Statin; dementia; risk; cognitive function; pathologic markers; meta-analysis

Submitted May 15, 2018. Accepted for publication Jun 21, 2018.

doi: $10.21037 / \mathrm{atm} .2018 .06 .43$

View this article at: http://dx.doi.org/10.21037/atm.2018.06.43

\section{Introduction}

Dementia is a complicated process, including the loss of synaptic connections, cell death, gliosis, and inflammation, as well as disruptions in functional networks, underlying cognition, behaviour, personality, and sensorimotor functions, eventually influencing an individual's autonomy (1). With the global population ageing rapidly, dementia has become a major public health problem. It affected approximately 
46.8 million people worldwide in 2015 , and the number of individuals with dementia will reach 131.5 million by 2050 (1). Considering the prevalence of dementia, it is critical to discover a useful method to diagnose and treat dementia.

A growing body of evidence suggests a close association between lipids and vascular changes in dementia. The betahydroxy-beta-methylglutaryl-CoA (HMGCoA) reductase inhibitor may reduce intracellular cholesterol/protein ratios and markedly inhibit beta-secretase cleavage of newly synthesised amyloid precursor protein in human HEK cells (2); in addition, the low-density lipoprotein (LDL) receptor-related protein receptor has been proven to play a possible role in Alzheimer's disease (AD) (3). Lipid-lowering agents (LLAs), particularly HMGCoA-reductase inhibitors (statins), may be helpful for certain arterial disorders (4-7), and these arterial factors are linked closely to dementia. Hence, LLAs may be a promising method to treat dementia. Current studies indicate that modulating lipid levels may influence dementia in elderly individuals; for example, two epidemiologic studies first reported that statin users may reduce the risk of dementia $(4,8)$. Furthermore, many research groups have begun to explore the effects of statins on dementia risk and pathologic changes (9-14). However, the U.S. Food and Drug Administration has asserted that there are several adverse effects of statin therapy, for example, confusion and memory loss in elderly people $(15,16)$.

Currently, statin therapy is a thriving area of research. However, as mentioned above, the current conclusions about statin therapy in dementia are incompatible. Although numerous studies have discussed the effects of statins on dementia, there is still a lack of studies providing an overview of their roles in dementia risk, cognitive changes, and pathologic changes. The existing articles discuss the role of specific statin therapies in specific types of dementia. Our study is the first to provide an overview of the various statin therapies in various types of dementias. We hope our study will provide investigators with more information about the progress of research on statin therapy for dementia. This paper is intended to review the currently available evidence to assess via meta-analysis the efficacy of statins for the treatment of dementia, including the effects of statin therapy on dementia risk, cognitive changes, and pathologic changes.

\section{Methods}

\section{Search strategy}

To assess the efficacy of statins for the treatment of dementia, including the effects of statin therapy on dementia risk, cognitive changes, and pathologic changes via meta-analysis, we searched for related literature in MEDLINE, EMBASE, the Cochrane Library, and BIOSIS previews for studies published in the period from January 1, 1987 to January 1, 2018. The search terms used are "dementia", "Alzheimer", "AD" and "statin" with Boolean operators as appropriate. We also obtained other relevant studies from meta-analyses and reference lists.

\section{Study selection}

Eligible studies for this meta-analysis met the following criteria: (I) the cohorts in the study were subjected to a period of statin therapy. Since the duration of most studies is inconsistent, we did not select specific durations; (II) the patients were diagnosed with dementia, $\mathrm{AD}$, vascular dementia $(\mathrm{VaD})$, or another type of dementia; (III) a variety of outcome measures for dementia were included, such as cognitive function, as measured by the assessments that follow. The mini-mental state examination (MMSE) is one of the most extensively and most frequently used cognitive tests, with high precision and accuracy. The total score for the MMSE is 30, and a higher score indicates better cognitive function. In addition, when a patient received a score of 23 or less, he or she was considered to suffer from cognitive impairment (17). The cognitive subscale of the Alzheimer's Disease Assessment Scale (ADAS$\operatorname{cog}$ ) is a testing method to measure cognitive changes in dementia-related drug trials (18). The ADAS-cog includes three items: descriptions, administrative procedures, and scoring. A higher score indicates better cognition function. The activities of daily life (ADL) scale is scored out of 100 and assesses basic self-care and mobility abilities. A higher score reflects greater independence (19). We also examined some pathological biomarkers, including plasma $\mathrm{A} \beta_{40}$, plasma $\mathrm{A} \beta_{42}$, cerebrospinal fluid (CSF) $\mathrm{A} \beta_{40}, \mathrm{CSF} \mathrm{A} \beta_{42}$, CSF total tau, CSF phosph-tau; plasma total cholesterol; plasma high-density lipoprotein (HDL) cholesterol; plasma LDL cholesterol; plasma triglycerides; plasma lathosterol; plasma 24S-hydroxycholesterol; CSF lathosterol, and CSF 24S-hydroxycholesterol. The included studies were required to contain the statistical information necessary to compute our results.

\section{Data extraction and quality assessment}

Zhu, Dai and Ma reviewed all appropriate articles according 


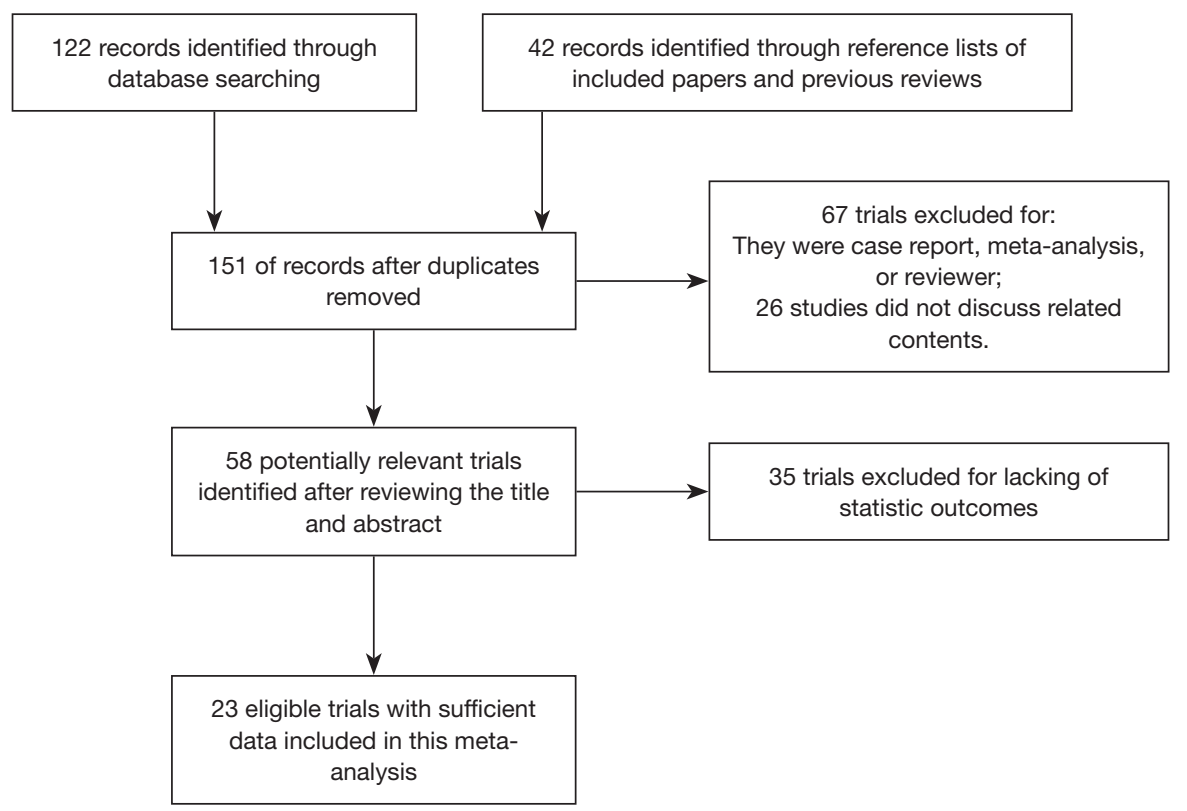

Figure 1 Flow diagram of study identification process.

to predefined criteria. We recorded the final valid statistics of each outcome by the mean, SDs, the number of patients at the time closest to the endpoint of the statin intervention, or the number of patients and controls with/without statin therapy. In addition, we also extracted the author name, data for country of study origin, published year, characteristics of participants (number, age, and number of males/females), and intervention details. When conflicts appeared in inclusion, exclusion, or data extraction, the conflicts were solved via discussion.

\section{Quantitative data synthesis}

Review Manager (version 5.2.3 for Windows, Copenhagen: The Nordic Cochrane Centre, The Cochrane Collaboration, 2012) was used to estimate the overall effects of statin therapy on dementia risk, cognitive changes, and pathologic markers by combining the results of each trial. Statistical heterogeneity was assessed using the $\mathrm{I}^{2}$ statistic. The results were considered heterogeneous when $\mathrm{I}^{2}$ was more than $50 \%$ (20). In this study, we chose a priori a random effects model for the outcome measures, sample characteristics, and heterogeneity of intervention types (21). When the number of methodologically sound studies was relatively small (less than 10), it was not necessary to use funnel plots to investigate publication bias. We evaluated publication bias only when the number of studies was more than ten (22).

\section{Results}

\section{Literature search and characteristics of included studies}

As the search strategy shows in Figure 1, a total of 23 relevant studies were included after the application of the search strategy. In total, 13 articles were included to explore the effects of statin therapy on dementia risk (4,23-34), and 11 studies were included to evaluate the effects of statin therapy on cognitive changes and pathologic changes (31,35-44). The details of the treatment methods were summarized in Table 1; specifically, these therapy methods are statin therapy (no mention of specific statin type) $(4,23-26,28-35,41$, 43-46), atorvastatin therapy $(4,27,40)$, fluvastatin therapy $(4,27)$, lovastatin therapy $(27,37)$, pravastatin therapy $(4,27,37)$, simvastatin therapy $(4,27,36-38,42,44)$, rivastigmin therapy (39), and cerivastatin therapy (4). We included general dementia patients (no mention of specific dementia type) (4,23-28,31-34), AD patients (24-26,29,30,35-38, $40-44)$, and $\mathrm{VaD}$ patients $(26,39)$ in this study.

\section{Effects of intervention}

\section{Dementia risk}

To reveal the effects of statin therapy on dementia risk, 


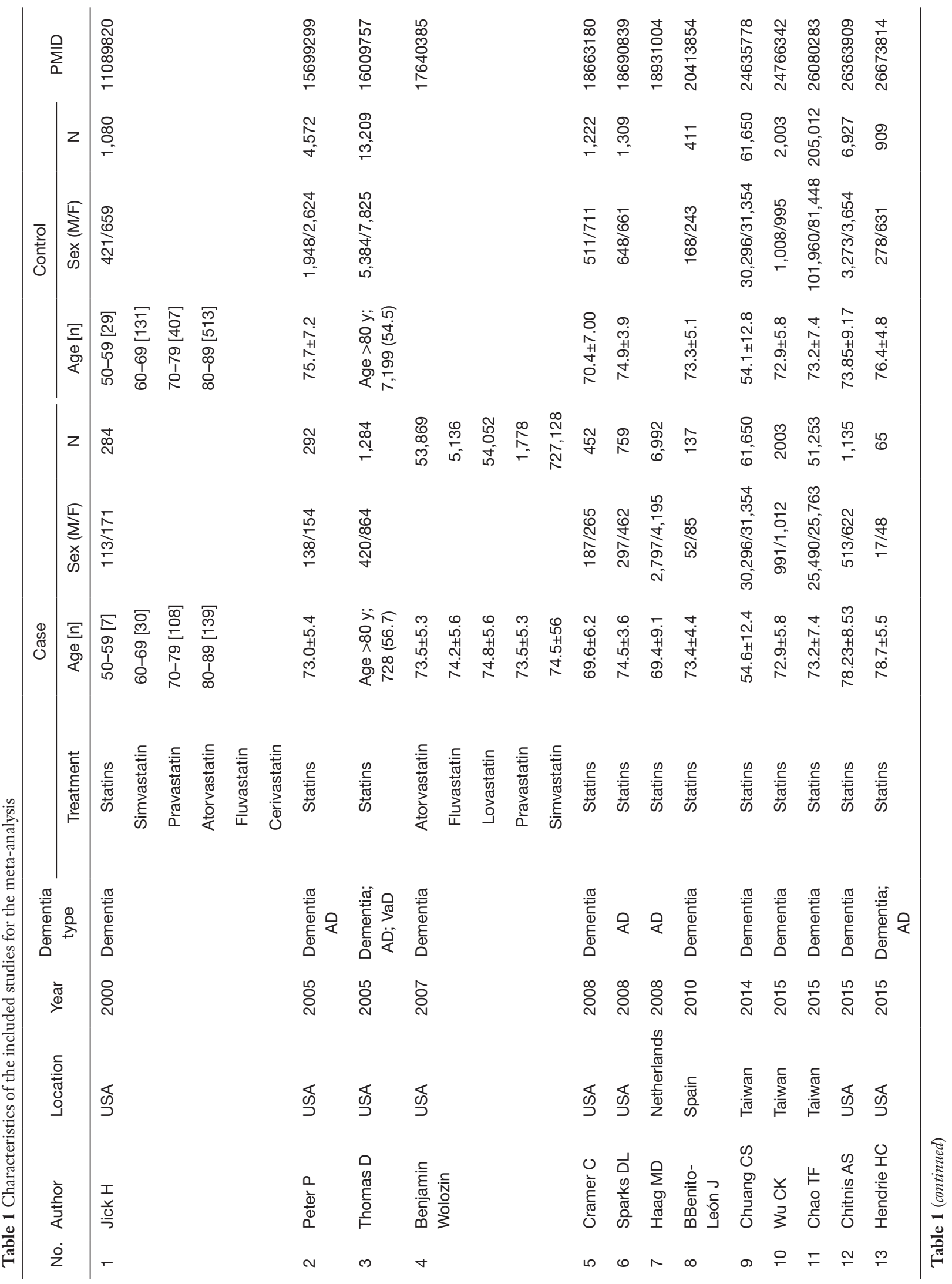




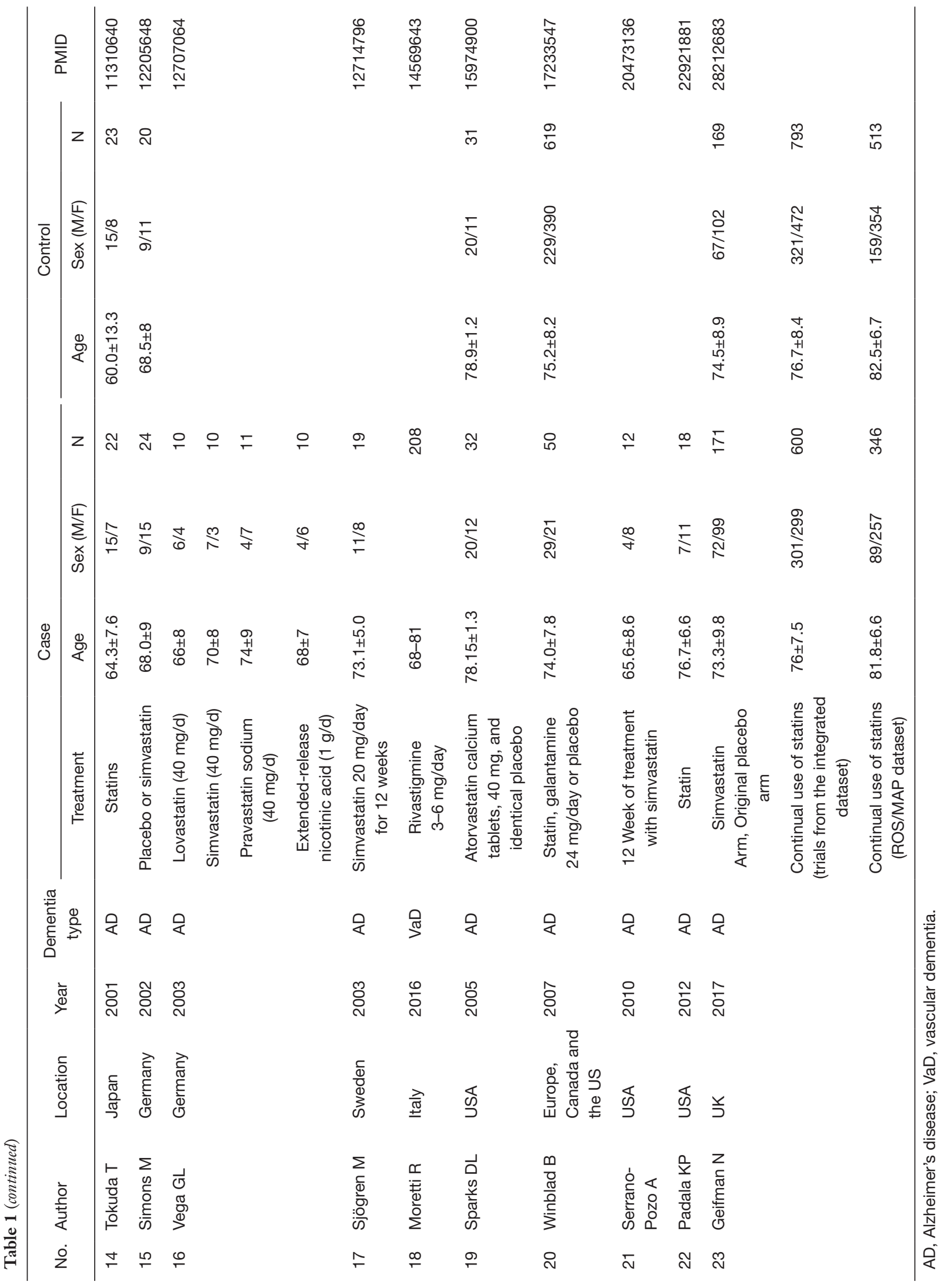




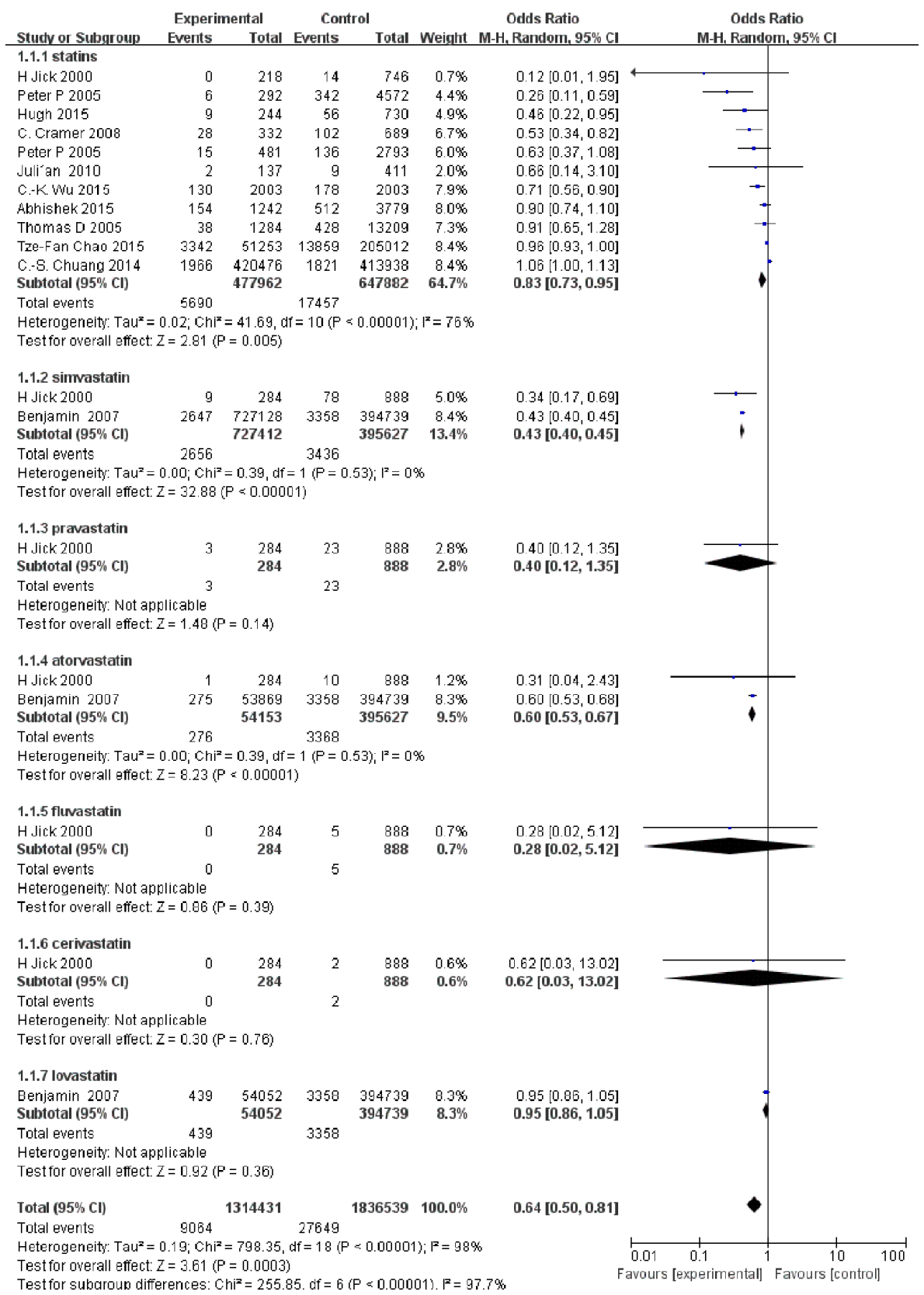

Figure 2 Forest plots show the effects of statin therapy on dementia risk.

we conducted a meta-analysis to assess the function of statin intervention on dementia risk, and the odds ratio $(\mathrm{OR})$ represented the modified ratio of incident dementia compared to the controls (Figure 2). Since the heterogeneity is $98 \%$, we used random effects model. In Figure 2, the pooled results show that statin therapy reduces dementia risk after analysing $1,314,431$ dementia patients and 1,836,539 healthy controls [OR: $0.64,95 \%$ confidence interval (CI): $0.50,0.81]$. When assessing specific types of statins, only two drugs, simvastatin and atorvastatin, have consistent results (OR: 0.43, 95\% CI: 0.40, 0.45; OR: 0.60, $95 \%$ CI: $0.53,0.67)$. The other four drugs mentioned, pravastatin, fluvastatin, cerivastatin, and lovastatin, showed no significant influence on dementia risk (OR: 0.40, 95\% CI: 0.12, 1.35; OR: 0.28, 95\% CI: 0.02, 5.12; OR: 0.62, 95\% CI: 0.03, 13.02; OR: 0.95, 95\% CI: 0.86, 1.05). 


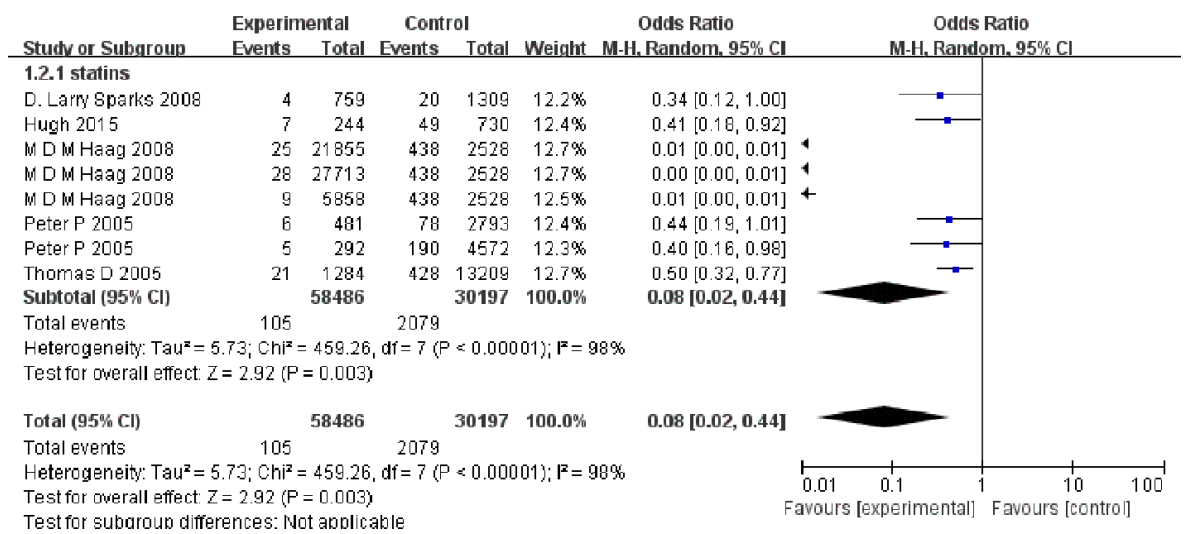

Figure 3 Forest plots show the effects of statin therapy on AD risk. AD, Alzheimer's disease.

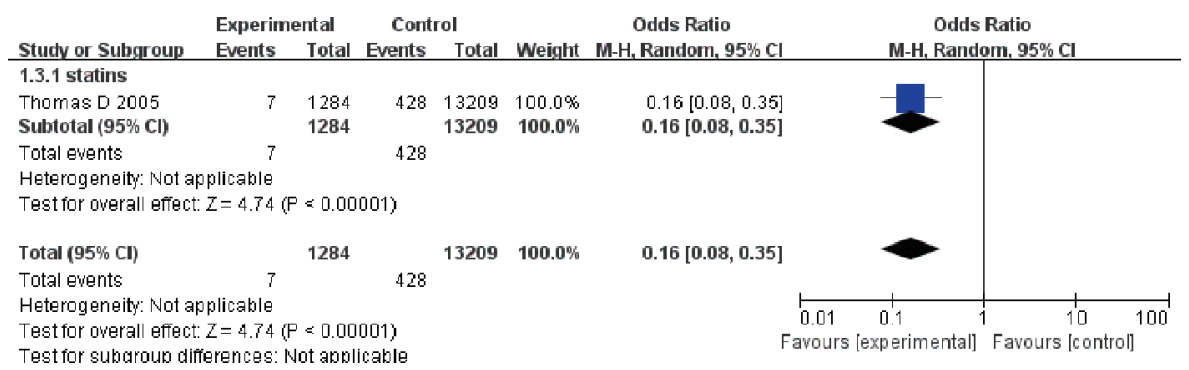

Figure 4 Forest plots show the effects of statin therapy on VaD risk. VaD, vascular dementia.

The results of our meta-analysis revealed statin therapy would reduce AD risk (OR: 0.08, 95\% CI: 0.02, 0.44) (Figure 3) in the random model used when heterogeneity is more than $50 \%$. VaD is another common dementia type. However, only one study of our included studies has concrete data to assess the pooled effect of statin therapies on $\mathrm{VaD}$ prevalence. This study indicated that statin therapy resulted in lower VaD prevalence (OR: 0.16, 95\% CI: 0.08 , 0.35 ) (Figure 4). These results may indicate that statin therapy meaningfully reduces the prevalence of the onset of dementia.

\section{Cognition}

Since MMSE score is a common measure to assess cognition function, our study tested the influence of statin therapy on cognition changes on the MMSE. Finally, our study indicated specific changes in MMSE score of individuals with dementia as a result of statin therapy (OR: $0.46,95 \%$ CI: $0.17,0.74$ ) (Figure 5A). The results indicated statin therapy may improve cognitive function. Of note, simvastatin intervention did not significantly alter MMSE score (OR: 0.70, 95\% CI: -2.23 , 3.63); however, atorvastatin therapy increased MMSE score
(OR: 0.57, 95\% CI: 0.23, 0.91).

ADAS-cog score is another common measure method to evaluate cognitive changes. However, inconsistent with MMSE score changes, the results of this meta-analysis showed statin therapy may not significantly alter the ADAScog score (OR: $-0.26,95 \%$ CI: $-1.33,0.62$ ) (Figure 5B).

\section{Activities of daily living (ADL)}

Only two studies were included to test the effects of statin therapy on ADL performance $(40,43)$, and the final results are shown in Figure 5. No significant association was observed between statin therapy and ADL performance (OR: -0.69, 95\% CI: -4.12, 2.74) (Figure 5C). Moreover, atorvastatin therapy significantly altered ADL performance (OR: $-2.40,95 \%$ CI: -3.33, -1.47) (Figure 5C).

\section{Pathological biomarkers}

A total of 16 items [plasma amyloid $\beta_{40}\left(\mathrm{~A} \beta_{40}\right)$, plasma $\mathrm{A} \beta_{42}$, CSF $\mathrm{A} \beta_{40}$, CSF $\mathrm{A} \beta_{42}$, CSF total tau, CSF phosphtau, plasma total cholesterol, plasma HDL cholesterol, plasma LDL cholesterol, plasma triglycerides, plasma lathosterol, plasma 24S-hydroxycholesterol, plasma 


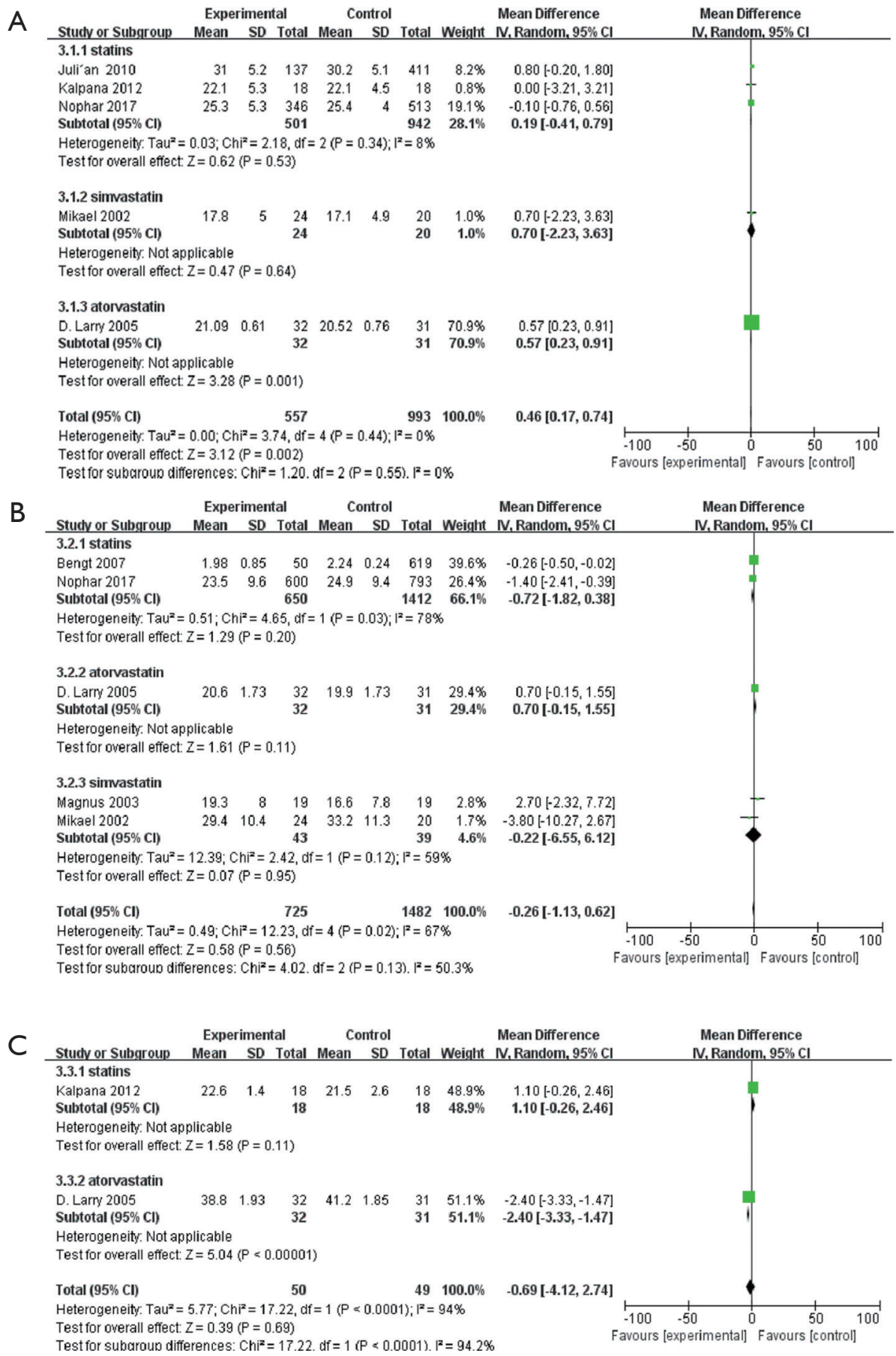

Figure 5 Forest plots show the effects of statin therapy on cognitive changes in dementia samples.

campesterol, plasma sitosterol, CSF lathosterol, and CSF 24S-hydroxycholesterol] were utilized to assess the effects of statin interventions on pathological biomarkers in dementia patients. As shown in Figure 6, the results indicate significant effects of statin therapy on plasma $\mathrm{A} \beta_{40}(\mathrm{OR}: 9.27,95 \% \mathrm{CI}$ :
$0.71,17.84)$ (Figure $6 A$ ), plasma $\mathrm{A} \beta_{42}(\mathrm{OR}: 2.60,95 \% \mathrm{CI}$ : $1.07,4.13)$ (Figure $6 B$ ), plasma LDL cholesterol (OR: -16.95 , 95\% CI: $-25.54,-8.37)$ (Figure 6E), plasma lathosterol (OR: $-0.11,95 \%$ CI: $-0.14,-0.07$ ) (Figure 6G), plasma 24s-hydroxycholesterol (OR: $-10.41,95 \%$ CI: $-15.57,-5.25$ ) 


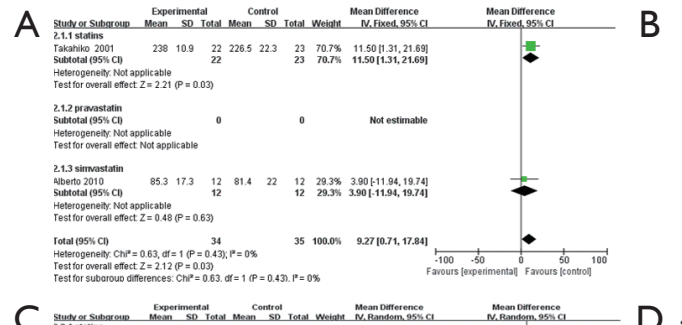

$\mathrm{C}$

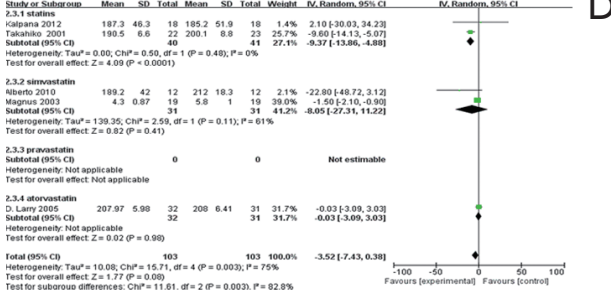

$\mathrm{E}$

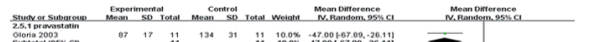

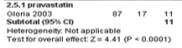

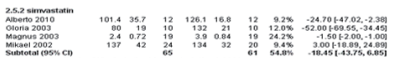

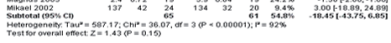

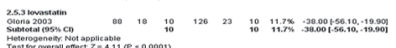

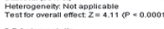

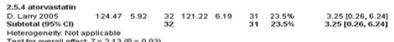

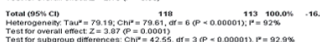

G

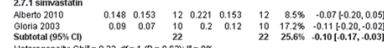

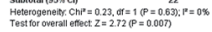

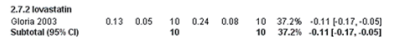

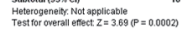

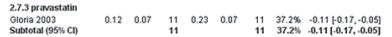

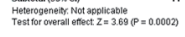

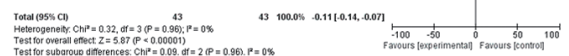

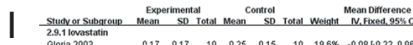

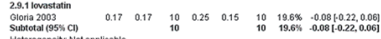

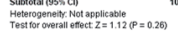

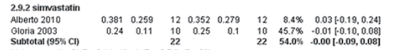

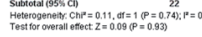

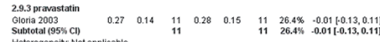

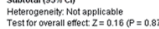

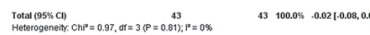

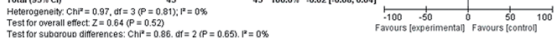

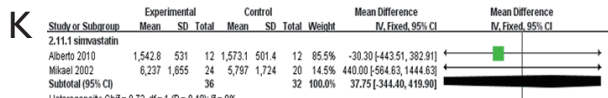

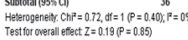

Toallasscon 36

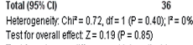

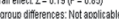

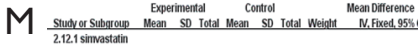

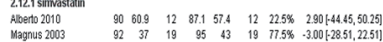

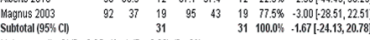

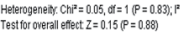

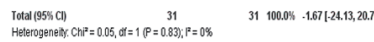

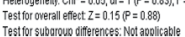

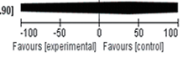

O

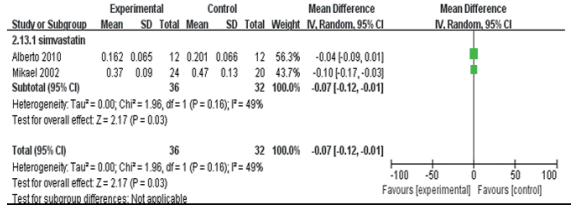

B

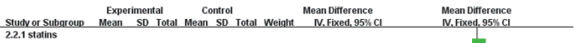

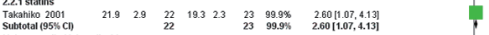

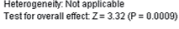

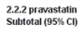

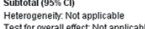

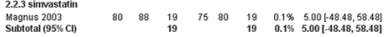

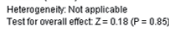

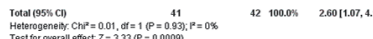

Trect $Z=333(p=0.00009)$

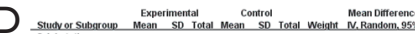

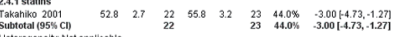

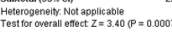

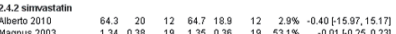

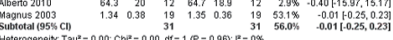

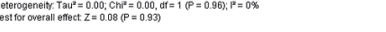

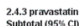

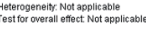

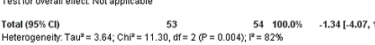

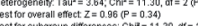

F

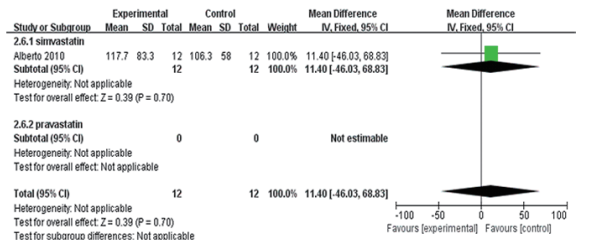

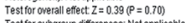

$\mathrm{H}$

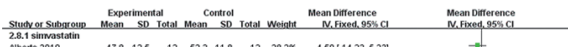

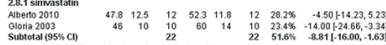

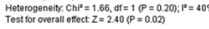

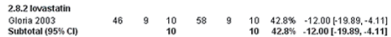

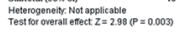

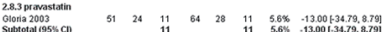

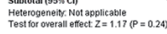

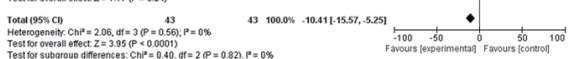

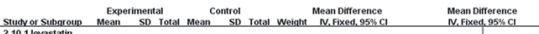

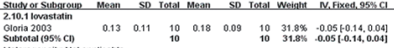

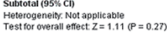

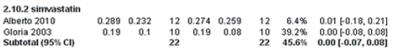

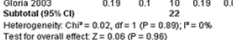

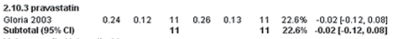

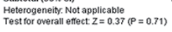

Totat (95:) CI)

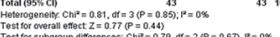

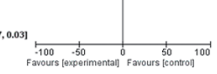

$\mathrm{L}$

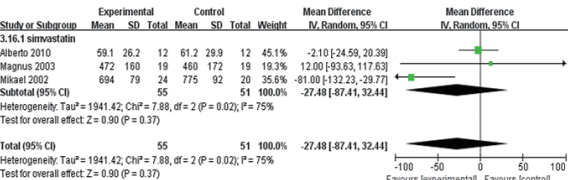

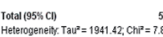

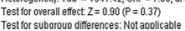

N

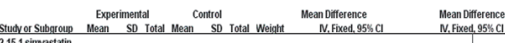

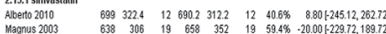

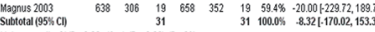

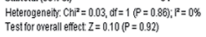

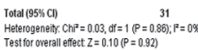

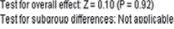

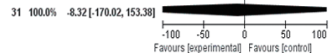

$\mathrm{P}$

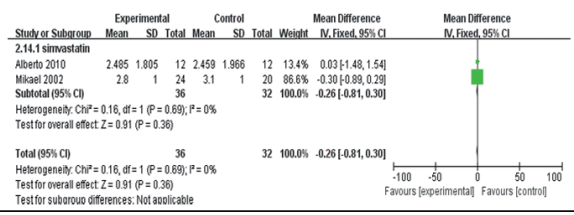

Figure 6 Forest plots show the effects of statin therapy on pathological markers in dementia samples. 
(Figure $6 H$ ), and CSF lathosterol (OR: $-0.07,95 \%$ CI: -0.12 , -0.01) (Figure 60). No significant results were observed for plasma total cholesterol (OR: $-3.52,95 \%$ CI: $-7.43,0.38$ ) (Figure 6C), plasma HDL cholesterol (OR: $-1.34,95 \%$ CI: -4.07, 1.39) (Figure 6D), plasma triglycerides (OR: 11.40, 95\% CI: -46.03, 68.83) (Figure 6F), plasma campesterol (OR: -0.02, 95\% CI: -0.08, 0.04) (Figure 6I), plasma sitosterol (OR: $-0.02,95 \%$ CI: $-0.07,0.03$ ) (Figure 67), CSF A $\beta_{40}$ (OR: 37.75, 95\% CI: $-344.40,419.90$ ) (Figure 6K), CSF $\mathrm{A} \beta_{42}$ (OR: $\left.-27.48,95 \% \mathrm{CI}:-87.41,32.44\right)$ (Figure $6 \mathrm{~L}$ ), CSF phospho-tau (OR: $-1.67,95 \%$ CI: $-24.13,20.78)$ (Figure 6M), CSF total tau (OR: $-8.32,95 \%$ CI: -170.02 , 153.38) (Figure $6 \mathrm{~N}$ ), and CSF 24S-hydroxycholesterol (OR: -0.26, 95\% CI: -0.81, 0.30) (Figure 6P).

\section{Publication bias}

Since the number of methodologically sound studies is relatively small (less than 10), it was not necessary to use funnel plots to investigate publication bias. In this study, we assessed publication bias via Begg's test. Accounting for the number of studies, we only explored publication bias as relating to the effects of statins on modulating dementia risk. Our results indicated no publication bias in for these studies overall in the meta-analysis.

\section{Discussion}

Higher cholesterol levels in mid-life are reported to increase the risk of developing $\mathrm{AD}$, and statin therapy may exert a protective influence against $\mathrm{AD}$ or dementia (16). Although these included articles showed inconsistent results about the effects of statin therapy on dementia, our study is an updated meta-analysis that evaluates the effects of statin therapy on dementia risk and pathological changes. Our study revealed that statin interventions would reduce dementia risk, especially decreasing $\mathrm{AD}$ and $\mathrm{VaD}$ prevalence. Statin interventions may improve MMSE score, and a significant role of statin therapy was observed in plasma $A \beta_{40}$, plasma $\mathrm{A} \beta_{42}$, plasma LDL cholesterol, plasma lathosterol, plasma 24s-hydroxycholesterol, and CSF lathosterol.

Vascular risk factors play critical roles in dementia progression; hence, modifying related factors may be a promising method to treat dementia. Interestingly, our study indicated that statin therapy may reduce dementia risk via modulating related pathologic biomarkers. Of note, statin therapy improves MMSE score, while not significantly altering ADAS-cog score. MMSE and ADAS-cog scores are two common cognitive assessments, but several differences exist between the two assessments. MMSE score is divided into two sections: the first part requires vocal responses and then covers orientation, memory, and attention; the second part tests the ability to name, follow verbal and written commands, write a sentence spontaneously, and copy a complex polygon (47). The ADAS-cog was designed as a rating scale to assess the severity of dysfunction in cognitive or non-cognitive behaviours in dementia patients. In addition, cognitive items and memory items account for approximately $60 \%$ of the total points (18). After comparing the two assessments, it is apparent that an ADAS-cog score reflects more abilities of memory. Consistent with the U.S. Food and Drug Administration's discovery, statin therapy may induce memory loss in elderly people. Therefore, statin therapy may increase abilities such as orientation, attention, naming, following verbal or written commands, and memory.

When discussing specific therapies, our results showed different results for different statin therapies. In this metaanalysis, six specific statin types were included, and they are simvastatin, pravastatin, atorvastatin, fluvastatin, cerivastatin, and lovastatin. In (online: http://atm.amegroups.com/ public/system/atm/supp-atm.2018.06.43-1.pdf), we provide the current research progress on these drugs in dementia. In addition, early biomarkers are crucial, and they may help to improve the diagnosis of dementia (48-50). These biomarkers include proteins in $\mathrm{A} \beta$ metabolism (51), tau metabolism (52,53), and lipid metabolism (54). Hence, we also evaluated the effects of statin therapy on dementiarelated biomarkers in Figure 6. Our results may be helpful in revealing the role of statin therapy in diagnosis and treatment of dementia.

As shown in (online: http://atm.amegroups.com/public/ system/atm/supp-atm.2018.06.43-1.pdf), simvastatin is a well-studied type of statin in dementia therapy. A dozen studies were carried out to explore its effect on dementia in clinical, in vivo, and in vitro studies. However, the results of clinical studies were inconsistent. After pooling these data, the results of our meta-analysis showed simvastatin may decrease dementia prevalence (OR: 0.43, 95\% CI: 0.40, $0.45)$, which indicated simvastatin therapy may alleviate related pathologic process. Although our group failed to find a close association in $\mathrm{A} \beta$ or tau metabolism, according to (online: http://atm.amegroups.com/public/system/atm/ supp-atm.2018.06.43-1.pdf), the in vivo and in vitro studies showed that simvastatin therapy may modulate $\mathrm{A} \beta$ (55-58) or tau metabolism $(55,59)$ by enhancing the phosphorylation of NR2B and Akt (60), altering the level of HIF-1 $\alpha$ and 
BACE (61), $\alpha 7$ nAChR-cascading the PI3K-Akt pathway, increasing BDNF (61,62), decreasing oxidative stress (63), blockading retinoblastoma protein phosphorylation, and inhibiting cyclin E/cyclin-dependent kinase (CDK) 2 activity associated with increased levels of the CDK inhibitors p21(Cip1) and p27(kip1) (64). Concerning lipid metabolism, we discovered that simvastatin therapy could downregulate the level of plasma 24s-hydroxycholesterol (OR: $-8.81,95 \%$ CI: $-16.00,-1.63$ ) and CSF lathosterol (OR: $-0.06,95 \%$ CI: $-0.10,-0.02)$. Moreover, the role of simvastatin therapy on lipid metabolism was proven in vivo and in vitro $(65,66)$. Considering the above studies, simvastatin therapy may be a promising method to treat dementia.

However, other statin therapies still lack sufficient data to prove their influence on dementia. Our group has even devoted our attention to lovastatin therapy. Our data indicate that statin-regulated sAPP secretion occurs via activation of the PI3K pathway and inhibition of ROCK signalling. Statins may modulate neuronal excite protection through both cholesterol-dependent and -independent mechanisms and maybe linked to calpain-mediated neuronal death (67). Our group demonstrated that lovastatin suppressed the aberrant tau phosphorylation both from frontotemporal dementia and Parkinsonism linked to a chromosome 17 (FTDP-17) mutation and okadaic acid induction in cultured rat primary neurons. The protective effect of lovastatin occurred at multiple pathological sites of tau protein, such as $\mathrm{Tyr}^{181}, \mathrm{Tyr}^{23} 1 \mathrm{Ser}^{202} / \mathrm{Tyr}^{205}, \mathrm{Tyr}^{212} / \mathrm{Ser}^{214}$ and $\mathrm{Ser}^{396} /$ $\mathrm{Ser}^{404}$ (68). In addition to our findings, a major study group also revealed similar results about the effects of lovastatin therapy on dementia-related pathologic changes, such as related to $A \beta$ (69-71). However, these current studies did not show its effects on dementia patients. It remains for more experimental groups to discover its true effects.

In summary, our meta-analysis offered some evidence of potential benefits of statin therapy on dementia. However, the major question is whether the current improvements are of clinical value. In addition, our study explored all types of study. In fact, there is low evidence in comparison to those based only on randomized controlled trials (RCTs). Therefore, further RCT trials with larger samples and longer interventions are needed to evaluate whether our findings are truly significant.

\section{Acknowledgements}

Funding: This work was supported by grants from the
National Natural Science Foundation of China [81000561], the Wuxi municipal health and Family Planning Commission Fund [Q201722], Wuxi Municipal Health Bureau found, Jiangsu basic research project (natural science foundation).

\section{Footnote}

Conflicts of Interest: The authors have no conflicts of interest to declare.

\section{References}

1. Elahi FM, Miller BL. A clinicopathological approach to the diagnosis of dementia. Nat Rev Neurol 2017;13:457-76.

2. Frears ER, Stephens DJ, Walters CE, et al. The role of cholesterol in the biosynthesis of beta-amyloid. Neuroreport 1999;10:1699-705.

3. Hyman BT, Strickland D, Rebeck GW. Role of the lowdensity lipoprotein receptor-related protein in betaamyloid metabolism and Alzheimer disease. Arch Neurol 2000;57:646-50.

4. Jick H, Zornberg GL, Jick SS, et al. Statins and the risk of dementia. Lancet 2000;356:1627-31.

5. Rosenson RS, Baker S, Banach M, et al. Optimizing Cholesterol Treatment in Patients With Muscle Complaints. J Am Coll Cardiol 2017;70:1290-301.

6. Gluba-Brzozka A, Franczyk B, Toth PP, et al. Molecular mechanisms of statin intolerance. Arch Med Sci 2016;12:645-58.

7. Banach M, Rizzo M, Toth PP, et al. Statin intolerance an attempt at a unified definition. Position paper from an International Lipid Expert Panel. Arch Med Sci 2015;11:1-23.

8. Wolozin B, Kellman W, Ruosseau P, et al. Decreased prevalence of Alzheimer disease associated with 3-hydroxy3-methyglutaryl coenzyme A reductase inhibitors. Arch Neurol 2000;57:1439-43.

9. Cicero AFG, Colletti A, Bajraktari G, et al. Lipid lowering nutraceuticals in clinical practice: position paper from an International Lipid Expert Panel. Arch Med Sci 2017;13:965-1005.

10. Zanchetti A, Liu L, Mancia G, et al. Blood pressure and LDL-cholesterol targets for prevention of recurrent strokes and cognitive decline in the hypertensive patient: design of the European Society of Hypertension-Chinese Hypertension League Stroke in Hypertension Optimal Treatment randomized trial. J Hypertens 2014;32:1888-97. 
11. Cicero AFG, Fogacci F, Banach M. Botanicals and phytochemicals active on cognitive decline: The clinical evidence. Pharmacol Res 2018;130:204-12.

12. Zanchetti A, Liu L, Mancia G, et al. Blood pressure and low-density lipoprotein-cholesterol lowering for prevention of strokes and cognitive decline: a review of available trial evidence. J Hypertens 2014;32:1741-50.

13. Ko D, Thigpen JL, Otis JA, et al. Influence of statin therapy at time of stroke onset on functional outcome among patients with atrial fibrillation. Int J Cardiol 2017;227:808-12.

14. Zissimopoulos JM, Barthold D, Brinton RD, et al. Sex and Race Differences in the Association Between Statin Use and the Incidence of Alzheimer Disease. JAMA Neurol 2017;74:225-32.

15. Everett BM, Smith RJ, Hiatt WR. Reducing LDL with PCSK9 Inhibitors--The Clinical Benefit of Lipid Drugs. N Engl J Med 2015;373:1588-91.

16. Banach M, Rizzo M, Nikolic D, et al. Intensive LDLcholesterol lowering therapy and neurocognitive function. Pharmacol Ther 2017;170:181-91.

17. Schultz-Larsen K, Lomholt RK, Kreiner S. Mini-Mental Status Examination: a short form of MMSE was as accurate as the original MMSE in predicting dementia. J Clin Epidemiol 2007;60:260-7.

18. Rosen WG, Mohs RC, Davis KL. A new rating scale for Alzheimer's disease. Am J Psychiatry 1984;141:1356-64.

19. Schiffczyk C, Romero B, Jonas C, et al. Appraising the need for care in Alzheimer's disease. BMC Psychiatry 2013;13:73.

20. Higgins JP, Thompson SG. Quantifying heterogeneity in a meta-analysis. Stat Med 2002;21:1539-58.

21. Field AP, Gillett R. How to do a meta-analysis. Br J Math Stat Psychol 2010;63:665-94.

22. Ioannidis JP. Interpretation of tests of heterogeneity and bias in meta-analysis. J Eval Clin Pract 2008;14:951-7.

23. Chitnis AS, Aparasu RR, Chen H, et al. Use of Statins and Risk of Dementia in Heart Failure: A Retrospective Cohort Study. Drugs Aging 2015;32:743-54.

24. Hendrie HC, Hake A, Lane K, et al. Statin Use, Incident Dementia and Alzheimer Disease in Elderly African Americans. Ethn Dis 2015;25:345-54.

25. Zandi PP, Sparks DL, Khachaturian AS, et al. Do statins reduce risk of incident dementia and Alzheimer disease? The Cache County Study. Arch Gen Psychiatry 2005;62:217-24.

26. Rea TD, Breitner JC, Psaty BM, et al. Statin use and the risk of incident dementia: the Cardiovascular Health
Study. Arch Neurol 2005;62:1047-51.

27. Wolozin B, Wang SW, Li NC, et al. Simvastatin is associated with a reduced incidence of dementia and Parkinson's disease. BMC Med 2007;5:20.

28. Cramer C, Haan MN, Galea S, et al. Use of statins and incidence of dementia and cognitive impairment without dementia in a cohort study. Neurology 2008;71:344-50.

29. Sparks DL, Kryscio RJ, Sabbagh MN, et al. Reduced risk of incident $\mathrm{AD}$ with elective statin use in a clinical trial cohort. Curr Alzheimer Res 2008;5:416-21.

30. Haag MD, Hofman A, Koudstaal PJ, et al. Statins are associated with a reduced risk of Alzheimer disease regardless of lipophilicity. The Rotterdam Study. J Neurol Neurosurg Psychiatry 2009;80:13-7.

31. Benito-Leon J, Louis ED, Vega S, et al. Statins and cognitive functioning in the elderly: a population-based study. J Alzheimers Dis 2010;21:95-102.

32. Chuang CS, Lin CL, Lin MC, et al. Decreased prevalence of dementia associated with statins: a national populationbased study. Eur J Neurol 2015;22:912-8.

33. Wu CK, Yang YH, Lin TT, et al. Statin use reduces the risk of dementia in elderly patients: a nationwide data survey and propensity analysis. J Intern Med 2015;277:343-52.

34. Chao TF, Liu CJ, Chen SJ, et al. Statins and the risk of dementia in patients with atrial fibrillation: A nationwide population-based cohort study. Int J Cardiol 2015;196:91-7.

35. Tokuda T, Tamaoka A, Matsuno S, et al. Plasma levels of amyloid beta proteins did not differ between subjects taking statins and those not taking statins. Ann Neurol 2001;49:546-7.

36. Simons M, Schwarzler F, Lutjohann D, et al. Treatment with simvastatin in normocholesterolemic patients with Alzheimer's disease: A 26-week randomized, placebocontrolled, double-blind trial. Ann Neurol 2002;52:346-50.

37. Vega GL, Weiner MF, Lipton AM, et al. Reduction in levels of 24S-hydroxycholesterol by statin treatment in patients with Alzheimer disease. Arch Neurol 2003;60:510-5.

38. Sjogren M, Gustafsson K, Syversen S, et al. Treatment with simvastatin in patients with Alzheimer's disease lowers both alpha- and beta-cleaved amyloid precursor protein. Dement Geriatr Cogn Disord 2003;16:25-30.

39. Moretti R, Torre P, Antonello RM, et al. Rivastigmine in subcortical vascular dementia: a randomized, controlled, open 12-month study in 208 patients. Am J Alzheimers Dis Other Demen 2003;18:265-72.

40. Sparks DL, Sabbagh MN, Connor DJ, et al. Atorvastatin therapy lowers circulating cholesterol but not free radical activity in advance of identifiable clinical benefit in the 
treatment of mild-to-moderate AD. Curr Alzheimer Res 2005;2:343-53.

41. Winblad B, Jelic V, Kershaw P, et al. Effects of statins on cognitive function in patients with Alzheimer's disease in galantamine clinical trials. Drugs Aging 2007;24:57-61.

42. Serrano-Pozo A, Vega GL, Lutjohann D, et al. Effects of simvastatin on cholesterol metabolism and Alzheimer disease biomarkers. Alzheimer Dis Assoc Disord 2010;24:220-6.

43. Padala KP, Padala PR, McNeilly DP, et al. The effect of HMG-CoA reductase inhibitors on cognition in patients with Alzheimer's dementia: a prospective withdrawal and rechallenge pilot study. Am J Geriatr Pharmacother 2012;10:296-302.

44. Geifman N, Brinton RD, Kennedy RE, et al. Evidence for benefit of statins to modify cognitive decline and risk in Alzheimer's disease. Alzheimers Res Ther 2017;9:10.

45. Bettermann K, Arnold AM, Williamson J, et al. Statins, risk of dementia, and cognitive function: secondary analysis of the ginkgo evaluation of memory study. J Stroke Cerebrovasc Dis 2012;21:436-44.

46. Steenland K, Zhao L, Goldstein FC, et al. Statins and cognitive decline in older adults with normal cognition or mild cognitive impairment. J Am Geriatr Soc 2013;61:1449-55.

47. Folstein MF, Folstein SE, McHugh PR. "Mini-mental state". A practical method for grading the cognitive state of patients for the clinician. J Psychiatr Res 1975;12:189-98.

48. Ahmed RM, Paterson RW, Warren JD, et al. Biomarkers in dementia: clinical utility and new directions. J Neurol Neurosurg Psychiatry 2014;85:1426-34.

49. Sancesario GM, Bernardini S. How many biomarkers to discriminate neurodegenerative dementia? Crit Rev Clin Lab Sci 2015;52:314-26.

50. Lu H, Zhu XC, Jiang T, et al. Body fluid biomarkers in Alzheimer's disease. Ann Transl Med 2015;3:70.

51. van Leijsen EMC, Kuiperij HB, Kersten I, et al. Plasma Abeta (Amyloid-beta) Levels and Severity and Progression of Small Vessel Disease. Stroke 2018;49:884-90.

52. Botha H, Mantyh WG, Murray ME, et al. FDG-PET in tau-negative amnestic dementia resembles that of autopsyproven hippocampal sclerosis. Brain 2018;141:1201-17.

53. Law LL, Rol RN, Schultz SA, et al. Moderate intensity physical activity associates with CSF biomarkers in a cohort at risk for Alzheimer's disease. Alzheimers Dement (Amst) 2018;10:188-95.

54. Kim WS, Jary E, Pickford R, et al. Lipidomics Analysis of Behavioral Variant Frontotemporal Dementia: A Scope for
Biomarker Development. Front Neurol 2018;9:104.

55. Carlsson CM, Gleason CE, Hess TM, et al. Effects of simvastatin on cerebrospinal fluid biomarkers and cognition in middle-aged adults at risk for Alzheimer's disease. J Alzheimers Dis 2008;13:187-97.

56. Li L, Cao D, Kim H, et al. Simvastatin enhances learning and memory independent of amyloid load in mice. Ann Neurol 2006;60:729-39.

57. Fassbender K, Simons M, Bergmann C, et al. Simvastatin strongly reduces levels of Alzheimer's disease beta -amyloid peptides Abeta 42 and Abeta 40 in vitro and in vivo. Proc Natl Acad Sci U S A 2001;98:5856-61.

58. Abrahamson EE, Ikonomovic MD, Dixon CE, et al. Simvastatin therapy prevents brain trauma-induced increases in beta-amyloid peptide levels. Ann Neurol 2009;66:407-14.

59. Dong W, Vuletic S, Albers JJ. Differential effects of simvastatin and pravastatin on expression of Alzheimer's disease-related genes in human astrocytes and neuronal cells. J Lipid Res 2009;50:2095-102.

60. Jin H, Chen T, Li G, et al. Dose-Dependent Neuroprotection and Neurotoxicity of Simvastatin through Reduction of Farnesyl Pyrophosphate in Mice Treated with Intracerebroventricular Injection of Abeta 1-42. J Alzheimers Dis 2016;50:501-16.

61. Jeong JH, Yum KS, Chang JY, et al. Dose-specific effect of simvastatin on hypoxia-induced HIF-1alpha and BACE expression in Alzheimer's disease cybrid cells. BMC Neurol 2015;15:127.

62. Mans RA, Chowdhury N, Cao D, et al. Simvastatin enhances hippocampal long-term potentiation in C57BL/6 mice. Neuroscience 2010;166:435-44.

63. Tuskova R, Liptak B, Szomolanyi P, et al. Neuronal marker recovery after Simvastatin treatment in dementia in the rat brain: in vivo magnetic resonance study. Behav Brain Res 2015;284:257-64.

64. Sala SG, Munoz U, Bartolome F, et al. HMG-CoA reductase inhibitor simvastatin inhibits cell cycle progression at the G1/S checkpoint in immortalized lymphocytes from Alzheimer's disease patients independently of cholesterol-lowering effects. J Pharmacol Exp Ther 2008;324:352-9.

65. El-Dessouki AM, Galal MA, Awad AS, et al. Neuroprotective Effects of Simvastatin and Cilostazol in L-Methionine-Induced Vascular Dementia in Rats. Mol Neurobiol 2017;54:5074-84.

66. Dias HK, Brown CL, Polidori MC, et al. LDL-lipids from patients with hypercholesterolaemia and Alzheimer's 


\section{Page 14 of 14}

disease are inflammatory to microvascular endothelial cells: mitigation by statin intervention. Clin Sci (Lond) 2015;129:1195-206.

67. Ma T, Zhao Y, Kwak YD, et al. Statin's excitoprotection is mediated by sAPP and the subsequent attenuation of calpain-induced truncation events, likely via rho-ROCK signaling. J Neurosci 2009;29:11226-36.

68. Li R, Xu DE, Ma T. Lovastatin suppresses the aberrant tau phosphorylation from FTDP-17 mutation and okadaic acid-induction in rat primary neurons. Neuroscience 2015;294:14-20.

69. Park IH, Hwang EM, Hong HS, et al. Lovastatin enhances
Zhu et al. Statin affects dementia risk and related pathologic change

Abeta production and senile plaque deposition in female Tg2576 mice. Neurobiol Aging 2003;24:637-43.

70. Buxbaum JD, Cullen EI, Friedhoff LT. Pharmacological concentrations of the HMG-CoA reductase inhibitor lovastatin decrease the formation of the Alzheimer betaamyloid peptide in vitro and in patients. Front Biosci 2002;7:a50-9.

71. Friedhoff LT, Cullen EI, Geoghagen NS, et al. Treatment with controlled-release lovastatin decreases serum concentrations of human beta-amyloid (A beta) peptide. Int J Neuropsychopharmacol 2001;4:127-30.
Cite this article as: Zhu XC, Dai WZ, Ma T. Overview the effect of statin therapy on dementia risk, cognitive changes and its pathologic change: a systematic review and meta-analysis. Ann Transl Med 2018;6(22):435. doi: 10.21037/atm.2018.06.43 